\title{
A unified solution to reduce test power and test volume for Test-per-scan schemes
}

\author{
Shaochong Lei, Zhen Wang, Zeye Liu, and Feng Liang ${ }^{\text {a) }}$ \\ School of Electronics and Information, Xi'an Jiaotong University, Xi'an, China, \\ 710049 \\ a) fengliang@mail.xjtu.edu.cn (corresponding author)
}

\begin{abstract}
This paper proposes a unified solution to reduce test power and test volume for test-per-scan schemes. With the self-testing using MISR and Parallel SRSG (STUMPS) architecture and the developed reconfigurable Johnson counter, the proposed test pattern generator (TPG) applies two transition sequences to all scan chains, and the primary inputs of the circuit under test (CUT) keep unchanged at most times. Therefore, the switching activities both in the combinational block and in scan chains can be reduced simultaneously. If the generated test vectors that do not contribute to fault coverage are filtered out, the remaining deterministic patterns show the favorable features of high compressible and low-test power. Simulation results on ISCAS'89 benchmarks demonstrate that the proposed TPG imposes negligible impact on test length and power overhead of the CUT.
\end{abstract}

Keywords: Test-per-scan, test pattern generation, low power, test compression

Classification: Integrated circuits

\section{References}

[1] M. Nourani, M. Tehranipoor, and N. Ahmed, "Low-transition test pattern generation for BIST-based applications," IEEE Trans. Comput., vol. 57, no. 3, pp. 303-315, March 2008.

[2] P. Girard, L. Guiller, C. Landrault, S. Pravossoudovitch, and H. J. Wunderlich, "A modified clock scheme for a low power BIST test Pattern generator," Proc. 19th IEEE Int. VLSI Test Symp., Marina Del Rey, California, USA 10.1109/VTS.2001.923454, pp. 306-311, April 2001.

[3] S. Wang and S. K. Gupta, "DS-LFSR: a BIST TPG for low switching activity," IEEE Trans. Computer-Aided Design Integr. Circuits Syst., vol. 21, no. 7, pp. 842-851, July 2002.

[4] S. Wang and S. K. Gupta, "LT-RTPG: a new test-per-scan BIST TPG for low switching activity," IEEE Trans. Computer-Aided Design Integr. Circuits Syst., vol. 25, no. 8, pp. 1565-1574, Aug. 2006.

[5] M. A. Gabarro, M. Lopez, J. Figueras, P. Girard, L. Guiller, C. Landrault, S. Pravossoudovitch, P. Teixeira, and M. Santos, "Low power BIST by filtering non-detecting vectors," Journal of Electronic Testing, vol. 16, no. 3, pp. 193-202, June 2000. 
[6] Z. Chen, S. C. Seth, D. Xiang, and B. B. Bhattacharya, "A unified solution to scan test volume, time, and power minimization," Proc. 23nd International Conference on VLSI Design, 10.1109/VLSI.Design.2010.105, Bangalore, India, pp. 9-14, Jan. 2010.

[7] N. N. Stelios and K. M. Maria, "Test set generation with a large number of unspecified bits using static and dynamic techniques," IEEE Trans. Comput., vol. 59, no. 3, pp. 301-316, March 2010.

[8] L. Li and K. Chakrabarty, "Test set embedding for deterministic BIST using a reconfigurable interconnection network," IEEE Trans. ComputerAided Design Integr. Circuits Syst., vol. 23, no. 8, pp. 1289-1305, Aug. 2008.

\section{Introduction}

Test-per-scan schemes are widely used in the sequential circuit testing. In these schemes, test patterns are first shifted into scan chains during the shift operation; test responses to these patterns are then captured in scan sells during the capture operation. The captured responses are shifted out to the output response analyzer (ORA) for response compaction. Because each test pattern requires a power-consuming shift operation to provide test patterns and evaluate test response, there are high switching activities in the combinational logic and in scan cells. This can cause excessive power dissipation. Studies show that the extra average or peak power consumption can create such problem as instantaneous power surge [1]. That may cause circuit damage, and reduces product yield and lifetime. There are many hardware approaches to reduce test power. Examples are LT-LFSR [1], the modified LFSR structure [2], DS-LFSR [3], LT-RTPG [4] and the two-vectorinhibiting TPG [5].

Another major drawback of the test-per-scan scheme is high test volume and long test time. For STUMPS-like scan architecture with multiple parallel scan chains, test volume and test time grow at a high rate with the size of the CUT [6]. That increases testing cost.

This paper proposes a novel test pattern generation method. In section 2 , it describes the structures of the seed circuit and the reconfigurable Johnson counter, and explains the principles of the proposed TPG. In section 3, it provides experimental methods and results on test power, fault coverage and power overhead of the proposed TPG, and compares it with the normal LFSR and some other TPGs. In section 4, it extends the proposed test generation method to test compression application. Finally, we give our conclusions in section 5 .

\section{Principle of the proposed TPG}

The proposed method utilizes the STUMPS-like scan architecture and the developed reconfigurable Johnson counter to generate low transition sequences. Fig. 1 (a) shows the general architecture of the proposed TPG. It consist of an $n$-stage normal LFSR with the primitive polynomial (the normal LFSR 
in brief), an $l$-stage Johnson counter and an XOR network. The inputs of the XOR network are taped from the outputs of the normal LFSR and the reconfigurable Johnson counter alternatively. If the numbers of their outputs are different, the number of two-input XOR gates in the network would be the smaller one. If the number of the primary inputs of the CUT is bigger than $n$, the outputs of the normal LFSR will be broadcasted to these primary inputs. Test responses can be compacted by a multiple input signature register (MISR). A normal LFSR is called a seed circuit, whose content at a time is called a seed.

Fig. 1(b) shows an example of the proposed TPG. Here, the number of the CUT's primary inputs, the number of scan chains and the maximal scan length are 7,4 and 7, respectively. The stage of the seed circuit and the stage of the reconfigurable Johnson counter will be selected as 4 and 7 , respectively. The outputs of the seed circuit $S_{1} S_{2} S_{3} S_{4}$ are broadcasted to the seven primary inputs of the CUT. Before testing applications, the seed circuit and the reconfigurable Johnson counter will be initialized. For our experiments in section 3 and section 4 , the initial values of all seed circuits are all one. If TPG_Mode is set to logic 1 and Init is set to logic 0 , the reconfigurable Johnson counter will remain in the all zero states after clocking CLK2 7 times. During the testing applications, test procedures of proposed TPG are as follows:

1). seed circuit generates a new seed by clocking CLK1 one time;

2). TPG_Mode is set to logic 0 , then CLK2 is clocked one time to generate one Johnson vector;

3). TPG_Mode and Init are set to logic 1, then CLK2 is clocked 7 times, and the outputs of the XOR network are shifted into scan chains 7 times;

4). a capture operation is inserted;

5). Repeat 2) until 14 Johnson vectors are generated;

6). Repeat 1)-5) until the expect fault coverage or test length is achieved.

Fig. 1 (c) shows the generated vectors applied to scan chains SC1-SC4 and the primary inputs, respectively. Here, the generated seed is $\{0111\}$, and the XOR networks will generate four SIC sequences for scan chains SC1-SC4. For example, for scan chain SC3, the applied sequences are $\{1110000\}$ and $\{1110001\}$ for two scan-in vectors. There are 1 or 2 transitions within these sequences. Generally, for an $l$-stage reconfigurable Johnson counter, vectors applied to each scan chain are some consecutive cyclic codewords of Johnson vectors. The cyclic codeword of a Johnson vector has one transition or two transitions. Also, the contents of a seed circuit keep unchanged during $2 l^{2}$ clocks of CLK2. Therefore, the mean input transition density of the combinational block of a scan design is less than $2 / l$ without considering capture operations. So, the proposed TPG can simultaneously reduce the transitions of the primary inputs and scan-in inputs. Test clocks and test control signals can be designed with the internal phase-locked loop (PLL), and the additional overhead is about 74 equivalent 2 -input gates. That imposes negligible area overhead to most CUTs. So we only analyze the performances of the proposed TPG. 


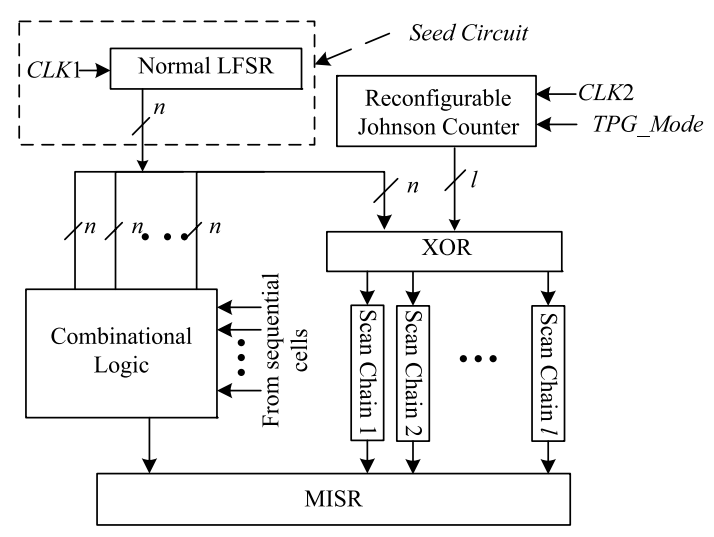

(a)

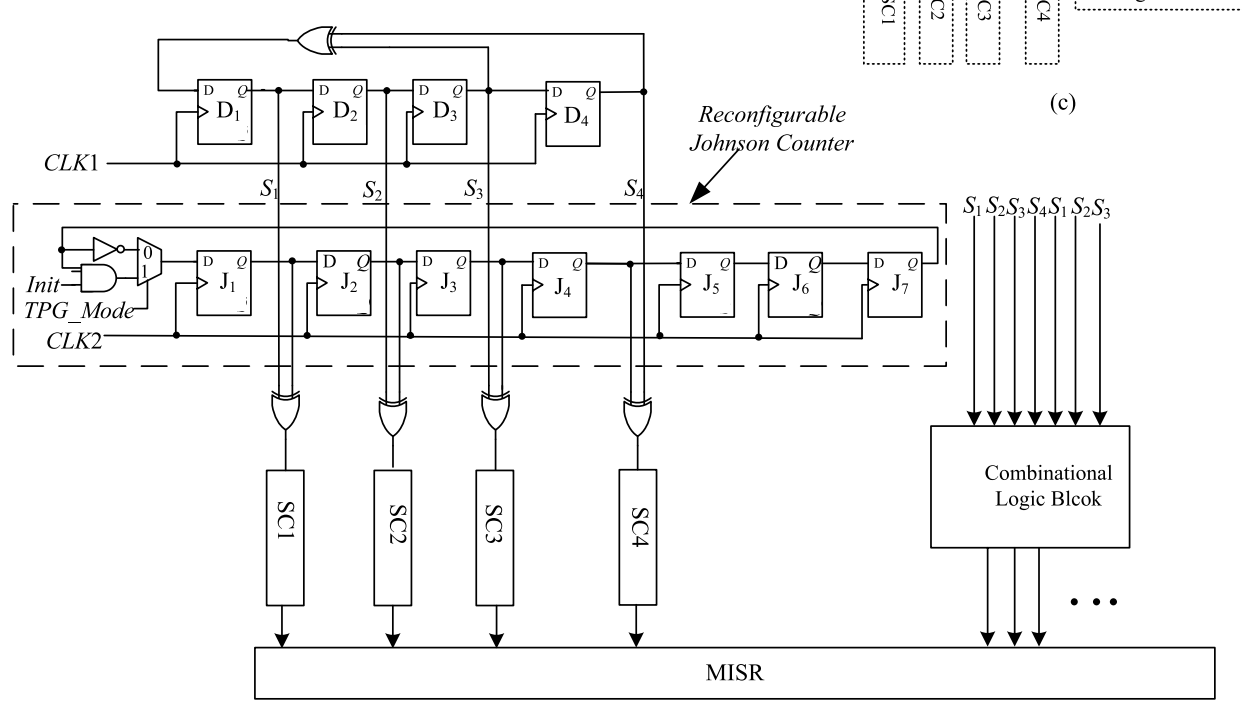

(b)

Fig. 1. (a) General architecture of the proposed TPG (b) An example of the proposed TPG (c) Some generated vectors for $(b)$

\section{Performance analysis of the proposed TPG}

To analyze the performance of the proposed TPG, experiments on full-scan designs of ISCAS'89 benchmarks are conducted. Performance simulations are carried out with Synopsys' Design Analyzer and Prime power. Fault simulations are carried out with Synopsys' Tetramax. Synthesis are carried out with SMIC $0.18 \mu \mathrm{m}$ technology library. The frequency of CLK2 is $100 \mathrm{MHz}$, and power supply voltage is $1.8 \mathrm{~V}$.

In Table I, columns 'TL', 'SFC' and 'TFC' refer to the test length, stuckat fault coverage and transition fault coverage, respectively. Sub-columns 'Prop.' and 'LFSR' refers to the simulation results with proposed TPG and with the normal LFSR, respectively. Sub-columns ' $\Delta$ avg.' and ' $\Delta$ peak' show average power reductions and peak power reductions with the proposed TPG against the normal LFSR, respectively. Power reductions ' $\Delta$ ' are calculated as $\Delta=\left(P_{L F S R}-P_{T P G}\right) / P_{L F S R} \times 100 \%$. Columns 'DFT Power $(\mathrm{mW})(\%)$ ' refer to the power of the proposed TPG and the corresponding percentage of the CUT's power with the normal LFSR. 
For all benchmarks except s38417 in Table I, the proposed TPG can achieve higher stuck-at fault coverage than the normal LFSR does. Also, the proposed TPG can test transition fault, and achieves higher coverage for three of the five largest ISCAS89's benchmarks. For s13207 and s38417, the transition fault coverage is lower with the proposed TPG than that with the normal LFSR. The main reason is that transition faults need longer test length. If the test length is 75000 , the transition fault coverage of s13207 will be $94.51 \%$ and $94.18 \%$ with the proposed TPG and with the normal LFSR, respectively. For s38417, the transition fault coverage is $96.14 \%$ and $94.96 \%$ with the proposed TPG and with the normal LFSR, respectively. The proposed TPG saves $42 \%-47 \%$ average power and $24 \%-52 \%$ peak power of the CUT against the normal LFSR. Compared with the TPG in [2], the proposed TPG can achieve higher stuck-at fault coverage, and the average power saving are approximative. The peak power saving vary a little. The average power of the proposed TPG is $1.8 \%-2.4 \%$ of that of the CUT, and the peak power of the proposed TPG is $1.3 \%-3.5 \%$ of that of the CUT. Therefore, the proposed TPG imposes negligible impact on average and peak power of the CUT.

Table I. Performance of the proposed TPG

\begin{tabular}{|c|c|c|c|c|c|c|c|c|c|c|c|c|}
\hline \multirow[t]{2}{*}{ Circuit } & \multirow[t]{2}{*}{ TL } & \multicolumn{2}{|c|}{$\begin{array}{l}\text { SFC } \\
(\%)\end{array}$} & \multicolumn{2}{|c|}{$\begin{array}{l}\text { TFC } \\
(\%)\end{array}$} & \multicolumn{2}{|c|}{$\begin{array}{l}\text { DFT Power } \\
(\mathrm{mW})(\%)\end{array}$} & \multicolumn{2}{|c|}{$\begin{array}{c}\text { Reduction } \\
(\%)\end{array}$} & \multicolumn{3}{|c|}{$\begin{array}{l}{[2]} \\
(\%)\end{array}$} \\
\hline & & $\begin{array}{l}\text { Prop. } \\
93.66\end{array}$ & $\begin{array}{c}\text { LFSR } \\
90.9\end{array}$ & $\begin{array}{l}\text { Prop. } \\
76.52\end{array}$ & $\begin{array}{l}\text { LFSR } \\
80.04\end{array}$ & $\begin{array}{c}\text { avg. } \\
0.3154(2.3)\end{array}$ & $\begin{array}{c}\text { peak } \\
4.852(3.0)\end{array}$ & $\begin{array}{c}\Delta \text { avg. } \\
45\end{array}$ & $\begin{array}{c}\Delta \text { peak } \\
52\end{array}$ & $\begin{array}{l}\text { SFC } \\
92.4\end{array}$ & $\begin{array}{c}\Delta \text { avg. } \\
48\end{array}$ & $\begin{array}{c}\Delta \text { peak } \\
44\end{array}$ \\
\hline s15850 & 9533 & 94.21 & 90.81 & 80.93 & 65.37 & $0.2736(2.2)$ & $5.133(3.5)$ & 44 & 32 & 90.61 & 44 & 47 \\
\hline s38417 & 9601 & 91.35 & 91.66 & 60.25 & 85.81 & $0.7377(1.8)$ & $4.961(1.3)$ & 45 & 47 & 91.75 & NA & NA \\
\hline s35932 & 4800 & 97.59 & 96.5 & 91.54 & 63.86 & $0.7844(2.4)$ & $5.255(1.4)$ & 42 & 24 & NA & NA & NA \\
\hline s38584 & 9645 & 97.16 & 94.24 & 89.92 & 80.01 & $0.8534(2.2)$ & $6.236(1.5)$ & 47 & 51 & 94.1 & 45 & 44 \\
\hline
\end{tabular}

\section{Application in test compression}

For the test vectors generated in section 2, if those do not contribute to fault coverage are filtered out, all remaining patterns contribute to fault coverage and can be easily compressed. Each compressed pattern is composed of the compressed Johnson vector and the seed. An l-stage Johnson counter can generates $2 l$ unique vectors, so these vectors can be compressed as $\left(\log _{2} 2 l+1\right)$ bit vectors. Studying column '\#Patterns' and column '\#Seed' in Table II, we can find that some test patterns share the same seed. Once the same seed has emerged in the previous compressed patterns, the following compressed patterns are only composed of the compressed Johnson vector. Therefore, if there are $N$ test patters that share $m$ seeds, the test compression ratio can be calculated as:

$$
\text { Ratio }=\frac{N \times(P I s+P P I s)}{m \times n+N \times\left(\text { integer }\left(\log _{2} 2 l\right)+1\right)}
$$

where PIs, PPIs and $n$ are the number of primary inputs, the number of scan cells and the width of the seed, respectively. For example, after 
simulating 65536 vectors of the proposed sequence as in section 2 for ISCAS'89 s13207, 428 test patterns can be determined. These test patterns show $98.24 \%$ stuck-at fault coverage, and share 225 unique seeds. The number of the primary inputs and the number of scan cells of ISCAS'89 s13207 are 62 and 638, respectively. The stage of the Johnson counter and the seed circuit are 64 and 31, respectively. Therefore, the compression ratio is

$$
\text { Ratio }=\frac{428 \times(62+638)}{225 \times 31+428 \times\left(\log _{2}(2 \times 64)+1\right)}=28.8
$$

Table II compares the compression ratios of the proposed method with those in $[6,7,8]$. Column 'Stage' refers to the stage of a Johnson counter or a seed circuit. Columns 'Ratio' refers to compression ratio. Sub-columns '\#Patterns' and '\#Seed' refer to the number of test patterns and the number of seeds, respectively. Compared with the method in [6], the proposed method demonstrates higher compression ratios. The proposed method can achieve much higher compression ratios and higher stuck-at fault coverage than the method in [8]. The proposed method also has much higher compression ratios than the method in [7].

Table II. Test volume compression ratio comparisons

\begin{tabular}{l|c|c|c|c|c|c|c|c|c|c} 
Circuit & PIs+DFFs & \multicolumn{2}{|c|}{ Stage (bit) } & \multicolumn{4}{|c|}{ Prop. } & \multicolumn{3}{c}{ Ratio } \\
\cline { 3 - 10 } & & Johnson & Seed & SFC (\%) & \#Patterns & \#Seed & Ratio & {$[6]($ SFC (\%)) } & {$[7]$} & {$[8]($ SFC (\%)) } \\
\cline { 3 - 10 } S13207 & $62+638$ & 64 & 31 & 98.24 & 428 & 225 & 28.8 & $10.88(98.46)$ & 13.69 & $4.28(96.5)$ \\
S15850 & $77+534$ & 54 & 39 & 97.46 & 486 & 267 & 20.8 & $9.46(96.68)$ & 4.85 & $4.56(95.9)$ \\
S38417 & $28+1636$ & 82 & 28 & 97.92 & 986 & 259 & 108.4 & $25.86(99.47)$ & 4.37 & $13.26(97.9)$ \\
S35932 & $35+1728$ & 87 & 35 & 99.97 & 274 & 21 & 165 & $41.85(89.90)$ & NA & NA \\
S38584 & $38+1426$ & 72 & 38 & 99.39 & 727 & 434 & 47.7 & $22.86(95.85)$ & 5.56 & $4.96(99.2)$ \\
\hline
\end{tabular}

\section{Conclusion}

To solve VLSI test problems in data volume, power and fault detection, this paper proposes a novel method to generate and compress test patterns. Unlike traditional test generation methods, the proposed method generates a class of test sequences that have linear relationship between their vectors. The predefined linear relationship is based on the single input change sequence. Implemented with a normal LFSR, a reconfigurable Johnson counter and an XOR network, the proposed method generate SIC sequences and two transition cyclic codewords for the CUT. That effectively reduces switching activities both in the combinational block and in scan chains of the CUT. Experimental results demonstrated that the proposed TPG can effectively reduce the average power and peak power of the CUT, and imposes negligible impact on test length. If the generated test vectors that do not contribute to fault coverage are filtered out, the remaining deterministic patterns show the favorable feature of high compressible. Therefore, the proposed method proposes a unified solution to reduce test power and test volume. 